\title{
Ockham y Wittgenstein. Acerca de los alcances y límites de la relación pensamiento-lenguaje ${ }^{* \dagger}$
}

\section{Ockham and Wittgenstein. On the Scope and Boundaries of the Thought-Language Relationship}

\section{Jean Paul Martínez Zepeda**}

\section{Resumen}

Para Ockham y Wittgenstein el análisis del conocimiento se articula en función del lenguaje. Ambos autores plantean la concepción de un mundo desde una dimensión lógica-filosófica configurada por la estrecha relación pensamiento-lenguaje. Construcción desarrollada en función de tres aspectos centrales: primero, los conceptos son signos de las cosas, segundo, las proposiciones describen "estados de cosas", y tercero, el conocimiento en cuanto "hábitos" es expresado en proposiciones articuladas en función de los "usos" del lenguaje, proposiciones establecidas por el pensamiento considerado como "actividad" y "operación de signos". En el análisis de determinadas proposiciones $a$ priori reconocemos los mismos límites de la relación pensamiento-lenguaje, en ellas se develan los problemas fundamentales del hombre, ya sea por la tendencia humana a la explicación del mundo como un todo o por la actitud del hombre frente al mismo.

Palabras clave: Ockham; Wittgenstein; signos; estados de cosas; hábitos; proposiciones.

\footnotetext{
* Recibido: 18/10/2018. Aceptado: 27/11/2018.

*** Pontificia Universidad Católica de Valparaíso, Chile. Email: jean.martinez.z@mail.pucv.cl

${ }^{\dagger}$ Agradezco al Prof. Doctor Mirko Skarica por sus comentarios al presente trabajo como su fundamental consejo en la elaboración de estos estudios.
} 


\begin{abstract}
For Ockham and Wittgenstein the analysis of knowledge is based on language. Both authors uphold the conception of the world from a logical-philosophical dimension configured by the close thought-language relationship. This construct is developed on the basis of the following three aspects: first, concepts are signs of things; second, propositions describe "state of affairs"; and third, knowledge in terms of "habits" is expressed in propositions structured in terms of the "uses" of language. These propositions are established by the thought considered as "activity" and "operation of signs". By analyzing certain a priori propositions, we recognize the very boundaries of the thought-language relationship, wherein the existential problems of mankind are revealed, whether out of the human tendency of explaining the world as a whole or out of the human attitude in the face of it.
\end{abstract}

Keywords: Ockham; Wittgenstein, signs; state of affairs; habits; propositions.

\title{
1. Introducción
}

Un aspecto fundamental que vincula las filosofías de Guillermo de Ockham y Ludwig Wittgenstein se encuentra en sus análisis acerca de la relación pensamiento-lenguaje. Ambos pensadores, en épocas y culturas diversas, abordan la solución de cuestiones filosóficas a partir del análisis de términos y proposiciones, situación que posibilita la exploración del conocimiento desde nuevas perspectivas que perfilan sus propios límites. En este campo, la pregunta por el conocimiento conlleva el detenido examen del lenguaje, posición que germinará, en las últimas etapas filosóficas de ambos autores, desde sus respectivas visiones sobre el estado del conocimiento como "conjuntos de hábitos" y asociados a los diversos "usos del lenguaje", análisis concordante con una concepción del pensamiento comprendido como "actividad" u "operación de signos".

En consideración de lo planteado el propósito del presente estudio consiste en explorar el análisis de la relación pensamiento-lenguaje en las obras de Ockham y Wittgenstein. De este modo, las interrogantes ¿por qué el concepto 
es un signo? ¿existe una relación entre proposiciones y estados de cosas? y ¿qué rol tienen los hábitos y usos del lenguaje al momento de comprender el conocimiento? orientan la comprensión de dicha relación la cual será articulada en función de tres hipótesis centrales:

Primero, los conceptos son signos. Para O. y W. los conceptos en cuanto signos son aprendidos en virtud del uso que les damos, esto es, en atención a las referencias que un grupo de hombres usa para describir los hechos del mundo. De este modo, en ambos autores se insiste que el significado guarda relación con la "referencia o denotación", en el caso de O. en virtud de la relación semántica entre conceptos, voces y palabras, y en W. desde el carácter significativo de los signos, posibilitado por el grado de referencia a los hechos y su despliegue a través del uso de términos y proposiciones que configuran nuestro mundo. En consecuencia, la cuestión planteada instala, en ambos filósofos, el "criterio de significación del lenguaje", constituido por el carácter semántico de los signos y su "uso" en virtud de su "denotación” a los singulares y los hechos.

Segundo, las proposiciones describen "estados de cosas" (state of affairs, Sachverhalt). O. y W. orientan la labor del análisis del lenguaje hacia la comprensión del sentido de nuestras proposiciones, es decir, en torno al despliegue del pensamiento. El "significado" de nuestros términos y proposiciones se establece a través del detenido examen de nuestros referentes a los singulares y sus relaciones en atención al uso convencional del lenguaje, situación que establece el rol de las proposiciones en la descripción de los hechos como "estados de cosas". Sin embargo, el análisis de las proposiciones nos conduce a reconocer la existencia de cierta clase de proposiciones a priori, las cuales conforman discursos bajo el anhelo humano de la explicación del mundo como un todo.

Tercero, el conocimiento es considerado como "hábitos" (habits) elaborados en correspondencia con los "usos" (uses) de términos y proposiciones. Cabe reconocer que el pensamiento como "actividad" considera la lectura del conocimiento en términos lingüísticos, esto es, desde la articulación del pensamiento en correspondencia con el desarrollo del lenguaje. Tanto para O. y W. el conocimiento como "conjuntos de hábitos" se expresa en "conjuntos de proposiciones" formuladas por la misma "actividad" del pensamiento como "operación de signos" (análisis y síntesis). De esta manera, el análisis 
del pensamiento comprende el examen del sentido de nuestras proposiciones, proposiciones que configuran nuestro mundo. Finalmente, y en virtud de las hipótesis planteadas, el presente trabajo se estructura en tres etapas: primero, el concepto, un signo. Segundo, proposiciones y "estados de cosas". Tercero, el conocimiento, "hábitos" y "usos" del lenguaje.

\section{El concepto, un signo}

El análisis de la relación pensamiento-lenguaje conlleva la atenta observación de los elementos que la posibilitan, en particular, la comprensión de los conceptos como signos de las cosas. En atención a esta problemática se propone indagar dos cuestiones fundamentales, por un lado, examinar la naturaleza del concepto como signo en $\mathrm{O}$. y por otro, analizar la lectura de W. en torno al signo en $\mathrm{O}$. Ahora bien, con el objeto de abordar estas cuestiones el presente apartado se articula en dos partes: primero, el concepto como signo natural en $\mathrm{O}$. y, segundo, análisis de la lectura de W. en torno al signo en $\mathrm{O}$.

En primer lugar, acerca del concepto como signo natural en O. El filósofo inglés mantiene la clásica división ya planteada por Boecio respecto a la clasificación de los términos en conceptos, voces y palabras (Ockham, 1978, 347-348). Los conceptos para O. son considerados signos naturales de las cosas, mientras que las voces y palabras, signos convencionales. Sin embargo, el franciscano, se distancia de una mirada clásica del concepto entendido desde la abstracción de esencias (Panaccio, 2004, 29-30) al sostener que el concepto es un signo que puede variar en cuanto el conocimiento intuitivo lo hace (Ockham, 1967, 30-33; 1970, 138-140), por tanto, la universalidad y certeza de nuestros conceptos no depende de un conocimiento inmutable circunscrito a esencias presentes formalmente en las cosas. Con su posición frente al concepto $O$. señala una nueva perspectiva acerca de la objetividad del conocimiento distante de formas por descubrir y circunscrita al conocimiento intuitivo de los singulares, el cual descansa en la denotación de los singulares contingentes (Panaccio, 2004, 12-13). De este modo, la predicación de los términos no depende de una esencia común sino de la extensión del concepto-signo el cual supone por conjuntos de singulares, predicación que pueda cambiar en la medida que los singulares y los hechos sobre los cuales significa lo hace. 
Por otro lado, el desarrollo de su teoría del lenguaje asume la comprensión de voces y palabras como "supuestos" de las cosas. La consideración de los términos como supuestos se articula en tres tipos de suposiciones personal, material y simple (Ockham, 1974, 195-197) las cuales configuran los modos en que los signos convencionales hacen referencias a distintos tipos de funciones en el lenguaje (Panaccio, 1999, 58). Toda la teoría de la suposición de O. se articula en atención a la significación de los términos y no al examen del campo psicológico de la abstracción de un concepto-esencia invariable que, para los realistas, se construye desde la atenta observación de la realidad mediante la contemplación del cosmos, las esencias son las fuentes de toda certeza durante el s. XIII.

Ahora bien, según lo señalado el concepto-signo para O. es, además, la ipsamet intellectio", es decir, la "misma intelección" adquirida por "costumbre"2, esto es, un "hábito" (Ockham, 1970, 291; 1986, 57). Cabe advertir que el concepto como intelección adquirida por hábitos es un signo natural ${ }^{3}$, un signo capaz de reconocer a través del lenguaje. De esta manera, la carga del contenido comprende el uso que orienta y determina el sentido de los térmi-

\footnotetext{
${ }^{1}$ Esta cuestión es expuesta por el Prof. Fortuny: “Esta ipsamet intellectio, esta ‘mismísima intelección', en acto permanece en el espíritu como cualidad mental en hábito, y puede ser reactivada con facilidad para su función lingüística de denotación y, ulteriormente, 'suposición"' (Ockham, 1986, 19). Además: “[...] como es lógico esperar, el concepto o signo mental para Ockham no es un verdadero conocimiento, suficiente por sí sólo. No hay un verdadero conocimiento a través de la vaciedad del signo -por muy natural que sea y muy denotativamente que funcione -sin su uso proposicional: sin que en la proposición, y sólo en ella, esté en lugar de suppongat la cosa conocida real. La significación no proporciona conocimiento sin la 'suposición': la frase simple o proposición es el primer conocimiento molecular mínimo" (Ockham, 1986, 19).

${ }^{2}$ Es importante señalar que la concepción empirista también reconoce la conexión entre el conocimiento como "hábitos y costumbres" y la lectura nominalista del lenguaje. Sobre el particular véase lo planteado por David Hume en su A Treatise of Human Nature (Hume, 2011, 46-49, 67). Una lectura sobre esta cuestión será examinada en otro estudio.

${ }^{3}$ Una fundamental explicación sobre el concepto en cuanto signo natural y hábito como su función semántica es expuesta por el Prof. Panaccio: "[...] that if a conceptual act is a caused in the right way by a certain habitus, then it inherits the semantical features of the act that had a caused (in the right way) this very habitus. Causal chains of the relevant kind must be semantically conducive" (Panaccio, 2004, 56) Además: "But the definition does inform us, at the same time, as the exact sense in wich a concept is said to be a sign in Ockham: in virtue of its causal history, it represents within the mind several individuals of the world such a way that it can stand for them in various mental propositional combinations, the true conditions of which it will then determinate in definitive ways. Ockham's distinctive nominalistic claim is that a semiotical system of this sort is indeed realized within the mind through causal chains of singular acts and habitus" (Panaccio, 2004, 58). Además, para una revisión sobre la naturaleza del concepto y sus implicancias en Ockham véase (Panaccio, 2004, 184-185).
} 
nos y proposiciones dentro de un sistema convencional. En este contexto, la distancia de $\mathrm{O}$. de una mirada clásica del lenguaje tiene su causa en la consideración de una incipiente relación pensamiento-lenguaje. El Profesor Panaccio, reconocido comentador de Ockham, ilustra esta cuestión a través del siguiente esquema de la significación natural y convencional en el pensador medieval (Panaccio, 1999, 55):

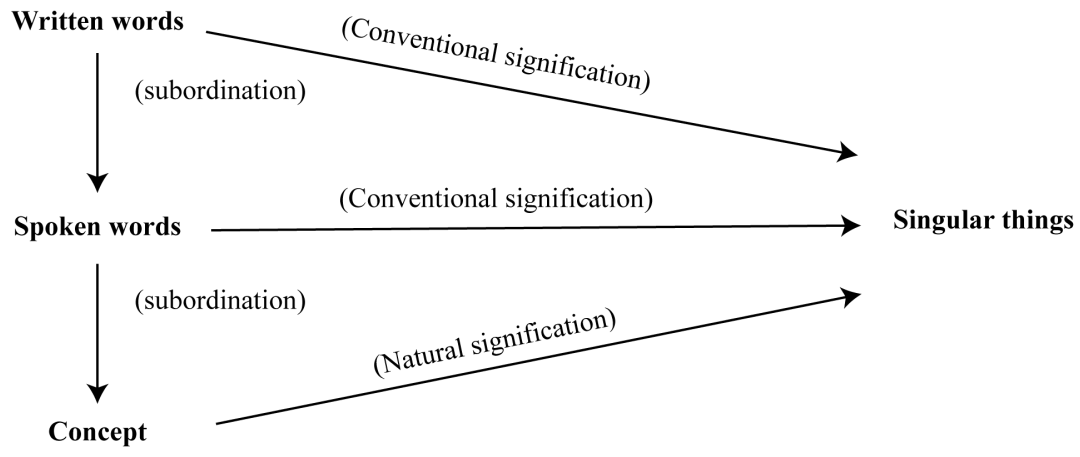

En segundo lugar, una relación entre $\mathrm{O}$ y W. se establece desde el análisis que W. ejecuta de la teoría del signo de $\mathrm{O}$. El autor de Tractatus logico-philosophicus al describir el signo utiliza la divisa reconocida como "principio de economía" de O. la cual manifiesta en los siguientes términos:

Un signo sólo determina una forma lógica si se le toma junto a su empleo lógico-sintáctico. (Wittgenstein, 2008a, 138).

Un signo que no se usa carece de significado. Este es el sentido de la divisa de Occam. (Si todo se conduce como si un signo tuviera significado, entonces tiene en efecto significado) (Wittgenstein, 2008a, 170)

Podemos inferir que la cuestión planteada aquí no es la mera exposición del "principio de economía" de los términos sino la lectura que $\mathrm{W}$. hace de la divisa, en particular, respecto al sentido que tendrían los signos para O. En este marco, el significado que atribuye W. a la divisa de O. y, de este modo, al sentido de los signos parece no dar cuenta de que su naturaleza se enlaza con la de los conceptos en el pensador franciscano. Los signos, para O. tienen un carácter semántico que obedece también a un contenido mental razón por la cual son denominados signos naturales. 
En consideración de lo expuesto, un problema fundamental nace al momento de analizar la posición de ambos autores respecto a la comprensión de los signos, en concreto, al reconocer que la cuestión incluye, en el caso de O. no sólo los signos convencionales, tales como voces y palabras, sino también la lectura del concepto como signo natural. W. parece omitir que la cuestión del signo natural planteada por O. determina el rumbo de toda su teoría de los signos. El vienés lee la teoría de los signos de O. en términos estrictamente convencionales, es decir, en cuanto determinados por el uso (Wittgenstein, 2008a, 138). La cuestión planteada por el autor del Tractatus consiste, por tanto, en la aceptación de un "criterio" de determinación del significado a través del uso, esta sería la idea que W. consideraría de O.

Cabe señalar que, en el caso del W. del Tractatus la concepción de los signos obedece a la denominada teoría "figurativa" en la que sostiene que el pensamiento versa sobre figuras, de este modo, la proyección figurativa es constitutiva del pensamiento en la medida que es determinada por el uso de los términos. Para O. en cambio, el signo natural orienta y determina el sentido de los signos convencionales, tal como lo plantea en la Suma de la Lógica (Ockham, 1974, 7-9).

No obstante, si bien es fundamental señalar que la teoría del signo "no es la misma" en $\mathrm{O}$. y W. en cuanto la teoría de la figuración del austríaco descansa en el carácter convencional del lenguaje, mientras que para $\mathrm{O}$. voces y palabras son convenciones subordinadas a los conceptos, divisamos en ambos autores una fecunda relación respecto al "modo" en que ambos enlazan los contenidos mentales al fundamental carácter semántico del lenguaje. O. establece el significado de los conceptos en la medida que hacen referencia a los singulares (Panaccio, 2004, 8, 50, 55). Para W. los signos son aprendidos a partir del uso que les damos, esto es, en virtud de las referencias que un grupo de hombres usa para describir los hechos del mundo, en ambos pensadores se insiste que el significado se halla en relación con la referencia a los singulares y los hechos. Por consiguiente, la cuestión planteada por ambos autores se ha de considerar en el campo del "criterio de significación del lenguaje", es decir, del carácter semántico de los signos como su uso en función de su referencia a los singulares y los hechos, este es el escenario común que ha de plantear el lenguaje entre todos los hombres, esto es lo constitutivo del pensamiento, la cuestión del significado. 
Además, conviene reconocer que el conocimiento intuitivo en O. posibilita una nueva comprensión del concepto (Ockham, 1967, 30-31) la cual se articula desde el punto de vista lógico en función de su denotación a conjuntos de singulares. Se dice que el concepto es un signo natural en cuanto es común por referencia y extensión a conjuntos de singulares, sin embargo, el significado de nuestros términos como signos naturales y convencionales para $\mathrm{O}$. pueden cambiar en tanto el conocimiento intuitivo de los singulares lo puede hacer. El significado, por tanto, descansa en último término en su referencia a singulares contingentes y los hechos en los que se conforma. De este modo, los significados de nuestros términos en $\mathrm{O}$. no obedecen a una esencia, en cuanto el concepto es la misma ipsamet intellectio, un hábito y en cuanto tal un signo. Dado lo anterior, son comprensibles las críticas de ambos autores al excesivo lenguaje de figuras abstractas presentes en la filosofía, ambos coinciden radicalmente en este punto: no es constitutivo del pensamiento lógico-filosófico el análisis a priori, no es constitutivo del pensamiento un lenguaje que no haga referencia a los singulares y sus relaciones, esta sería la lectura común que entregaría la divisa planteada ya en el S.XIV por O.

Cabe añadir, por otro lado, que desde el punto de vista semántico O. establece una central diferencia respecto a la propiedad de los términos manifestada en su distinción entre categoremáticos y syncategoremáticos. En el caso de los categorématicos reconocemos la función que tienen los términos para hacer referencia directa a los singulares, tal es el caso de los términos "hombre" o "árbol" los cuales suponen directamente por clases de singulares (Panaccio, 1999, 58). Por otro lado, los syncategoremáticos, son aquellos términos que cobran significado en relación a los categoremáticos para la formulación de proposiciones, ejemplos de ellos son las conjunciones y preposiciones: "y", "o", "porque", "entonces", etc. (Ockham, 1974, 40, 735, 798). Así pues, debemos precisar que en el caso de los syncategoremáticos la cuestión de la referencia ${ }^{4}$ reconoce la articulación de la función de distintos

\footnotetext{
${ }^{4}$ Según Panaccio, los syncategoremáticos: “[...] are used in propositional contexts to determinate the modes of reference of names and verbs and the logical structure of the propositions" (Panaccio, 1999, 55). Por otro lado, respecto a la estructura proposicional y su referencia a los singulares y su disposición y configuración en distintos órdenes Panaccio sostiene: "The whole structure of propositional discourse is ultimately brought to rest, for its truth and falsity, upon the ways in which the various singular beings of the world are arranged with regard to each other along certain ordered series, such as the causal, the temporal, and the local ones. The orderings themselves, though, are denied any independent reality" (Panaccio, 1999, 63) Sin embargo, frente a la independencia real de tales órdenes el Prof. Paul Vincent Spade aclara:
} 
tipos de términos (Panaccio, 2004, 154), los cuales son comprendidos desde su uso para la formulación de proposiciones que posibilitan la comprensión y análisis de los hechos, proposiciones que mediante el juicio pueden ser consideradas como verdaderas o falsas ${ }^{5}$. En este sentido, podemos interpretar que el conocimiento del significado de términos y proposiciones, en el contexto de la institución humana, presupone el "dominio" de un lenguaje aprendido desde la conexión entre términos categoremáticos y syncategoremáticos. Por tanto, la problemática del pensamiento en O. no sólo incluye el análisis de los conceptos en virtud de sus referencias ${ }^{6}$ a los singulares en cuanto signos naturales sino, además, el eminente carácter de la convención en la articulación de signos para la configuración de proposiciones ${ }^{7}$ en las cuales se expresa la relación pensamiento-lenguaje.

Ahora bien, en el caso de W. el problema de la referencia se mantiene en su lectura de los términos como signos, los cuales operan y cobran sentido en la proposición:

A estos elementos los llamo "signos simples" y a la proposición "completamente analizada". (Wittgenstein, 2008a, 129)

\footnotetext{
"What Ockham does is not to deny 'all' reality to such orderings and other truth-relevant factors; instead, he merely denies they are 'things'. In practice, this amounts to denying only that they are factors that can be signified, and so in particular that they can be signified by absolute categorematic terms" (Spade, 1999, 108).

${ }^{5}$ En palabras de Ockham: "Sed veritas et falsitas sunt quaedam praedicabilia de propositione, importantia quod ita est a parte significati sicut denotatur per propositionem quae est signum; unde propositionem esse veram non est propositionem habere aliquam talem qualitatem in se sed propositionem esse veram est ita esse sicut significatur per propositionem. Unde si Sortes sedet, tunc haec est vera 'Sortes sedet', quia ita est sicut denotatur per istam propositionem 'Sortes sedet'; et si 'Sortes non sedet', tunc haec est falsa 'Sortes sedet', quia non est ita sicut denotatur per istam 'Sortes sedet'; et eodem modo omnibus aliis est dicendum" (Ockham, 1978, 376). Además, sobre el rol de la composición y división en el juicio véase (Ockham, 1978, 373). Sobre los actos judicativos véase (Panaccio, 2004, 36-35)

${ }^{6}$ Los distintos tipos de referencias son analizados por Ockham desde los diversos modos de suposición de los términos al momento de reconocer los singulares y su rol dentro de las proposiciones que describen los hechos. En este contexto, debemos afirmar que la lógica de Ockham no se realiza en el marco de un análisis de "fenómenos mentales" sino más bien se amplía a la exploración las funciones y usos que hacemos de los términos y proposiciones, en particular, al momento de comprender los singulares y sus relaciones desde la fundamental correspondencia entre pensamiento y lenguaje. Tal reflexión es posible reconocer en torno al mismo análisis del concepto que realiza Ockham: "Qualis autem sit ista passio, an scilicet sit aliqua res extra animan, vel aliquid realiter exsistens in anima, vel aliquod ens fictum exsistens tantum in anima obiective, non pertinet ad logicum sed ad metaphysicum considerare" (Ockham, 1978, 349).

${ }^{7}$ Una lectura sobre el rol de los syncategoremáticos y las constantes lógicas en Ockham y Wittgenstein se encuentra en (Panaccio, 2004, 145).
} 
Los signos simples empleados en las proposiciones se llaman nombres. (Wittgenstein, 2008a, 129)

A una configuración de signos simples en un signo proposicional le corresponde una configuración de objetos en una situación. (Wittgenstein, 2008a, 129)

Vemos que en la posición de W. los signos sirven en la proposición en cuanto expresan, esto es, en cuanto hacen referencia a "estados de cosas". Caso semejante encontramos en O. para quien los términos en cuanto signos cobran sentido en la proposición mediante la expresión de los singulares y sus relaciones, los signos sólo expresan en la proposición, esto configura nuestro conocimiento. Para O. y W. la fuerza de la denotación del signo ha de operar en virtud de la descripción, esto es lo que para W. configura el pensamiento (Wittgenstein, 2008a, 129). He aquí el valor de las teorías semánticas de ambos autores, los cuales mediante la descripción de los hechos reconocen distintos órdenes de la realidad, posición que nos proyecta a concebir la referencia o "denotación" en relación con la predicación de nuestros términos en general (Ockham, 1974, 47-49). La proposición, por tanto, constituye el conocimiento, el cual es posible determinar desde el análisis del significado de términos y proposiciones y no desde esencias, tal como lo entendían los escolásticos del S.XIII, posición manifiesta en su esfuerzo por el descubrimiento de la forma del mundo a partir de nociones puras. Para O. la cuestión no será la explicación teórica del mundo sino la paulatina descripción de hechos expresados por el mismo lenguaje. En definitiva, O. y W. nos muestran un mundo semántico en que los signos pensados son los signos expresados en la proposición, mundo que analizaremos, a continuación, desde la estrecha relación entre proposiciones y su referencia a "estados de cosas".

\section{Proposiciones y "estados de cosas"}

Se ha sostenido en el apartado anterior que la distancia de la concepción de un conocimiento esencial como fuente de toda objetividad y certeza de nuestras proposiciones configura una perspectiva distinta del pensamiento, la cual descansa en un protagonismo incipiente del sujeto que conoce los hechos. En este campo, toda articulación de estructuras que impongan una determinada forma de pensar los hechos constituye un pre-juicio sobre lo que llamamos 
mundo, tal es el caso de doctrinas metafísicas que, por lo general, atribuyen a los términos significados cristalizados, los cuales pretenden ir más allá de los "estados de cosas". En este ámbito, O. y W. orientan la labor del análisis del lenguaje hacia la comprensión del sentido de nuestras proposiciones, es decir, en torno al despliegue del pensamiento. El "significado" de nuestros términos se establece a través del detenido examen de nuestros referentes a los singulares y sus relaciones. Esta situación se extrapola, además, al campo de las proposiciones y su rol en la descripción de los hechos como "estados de cosas". En consideración de estos elementos el presente apartado ejecuta dos análisis: primero, acerca de las proposiciones y "estados de cosas", y segundo, respecto a la relación pensamiento-lenguaje en O. y W.

Primero, acerca de las proposiciones y "estados de cosas". Según lo expuesto, un crucial punto de encuentro en $\mathrm{O}$. y W. es el rol que tienen las proposiciones en la formulación del conocimiento, cuestión que nos permite establecer una semejanza de consideración entre estos pensadores al momento de comprender el estatuto de las proposiciones que constituyen el pensamiento.

Desde la reflexión de O. podemos sostener que la lectura de las proposiciones en términos de la expresión de "estados de cosas" establece un aspecto crucial al momento de abordar un conocimiento significativo de conceptos y no un conocimiento universal de esencias, esta perspectiva plantea el necesario examen del conocimiento en el campo del análisis de términos y proposiciones, posición nuclear al momento de concebir el lenguaje como conjuntos de signos donde la verdad o falsedad del enunciado depende sólo de los hechos, es decir, de su correspondencia con "estados de cosas". Sobre el particular, De Andrés reconoce la cuestión de las proposiciones y su relación con "estados de cosas" en O. lo cual expone en los siguientes términos: "[...] lo que justifica, pues, y constituye la verdad de la proposición para O. es el hecho de que sea una misma cosa aquella por la que supone el sujeto y por la que supone el predicado. Esa identidad es el 'estado de cosas' connotado por la verdad de la proposición” (De Andrés, 1969, 269).

En este sentido, para O. la cuestión fundamental consiste en reconocer que el conocimiento evidente sólo es posible en torno a las proposiciones, según el pensador medieval: "[...] que toda ciencia, sea real o racional, es tan sólo de las proposiciones como objetos que se saben, pues sólo se saben las 
proposiciones" (Ockham, 1970, 134-138; Fernández, 1980, 1018). O. perfila su comprensión de las proposiciones en la medida que versan sobre conocimientos en directa relación con el estado de los singulares, en definitiva, no hay demostración sin el conocimiento intuitivo de los singulares ${ }^{8}$. Sobre el particular, es posible comprender que en el caso de O. la cuestión de las proposiciones universales implica la negación de su referencia a esencias como base de la validez universal de los juicios, para el pensador inglés, por tanto, no es posible establecer proposiciones "invariables" de las cuales dependa una explicación general del mundo. El carácter significativo de nuestras proposiciones descansa en su referencia a "estados de cosas" posibles.

Respecto a la posición de W. la cuestión del "estado de cosas" constituye un aspecto central que posibilita la comprensión lógica de la verdad de las proposiciones, la cual establece la formulación de conjuntos de proposiciones que nos muestran el mundo: "[...] la totalidad de los pensamientos verdaderos es una figura del mundo" (Wittgenstein, 2008a, 124). Sobre este tema el Tractatus de W. plantea dos aspectos fundamentales.

Por un lado, "No hay figura alguna que sea verdadera a priori" (Wittgenstein, 2008a, 124). Para W. las verdades a priori corresponden a meta-figuras de las cuales no es posible obtener una evidencia comparable a las proposiciones que figuran los hechos del mundo. De este modo, para el pensador austríaco, sólo la figuración de los hechos del mundo constituye pensamiento, lo cual sostiene al afirmar: "Una figura lógica de los hechos es un pensamiento" (Wittgenstein, 2008a, 124). Las proposiciones describen y tienen sentido en la medida que corresponden a "estados de cosas", el significado queda establecido en el modo apropiado en que las palabras nos muestran los hechos del mundo. Sobre el particular, Kenny señala que la relación abordada entre proposición y situación "no es casual sino interna" y que la verdad o falsedad de la proposición depende de su comparación con la realidad para

\footnotetext{
8 "Dico quod obiectum motivum intellectus est praecise singulare. Et dico quod omne singulare est motivum intellectus, quia omne singulare potest intelligi notitia intuitiva, quantum est ex naturae animae et intellectus nostri" (Ockham, 1970, 540) "[...] quia complexum de singulari non est principium demostrandi, et tamen sine illo non potest haberi demostratio, et illa Semper notitia singularis pressuponitur" (Ockham, 1970, 503).

${ }^{9}$ Sin embargo, según Kenny, es necesario señalar que: “[...] aunque la proposición pueda representar el estar acostado el gato en el felpudo, no puede representar lo que hay en común entre esta situación y ella misma, a saber, la forma lógica (TLP 4.12). La forma lógica se refleja en las proposiciones, no la representan ellas; es decir, la muestran ellas y ellas la poseen (TLP 4.21)" (Kenny, 1988, 68)
} 
describir un estado de cosas real (Kenny, 1988, 68-69). En este ámbito el mismo W. afirma: "El signo proposicional consiste en que sus elementos, las palabras, se relacionan unos con otros de modo y manera determinados. Un signo proposicional es un hecho" (Wittgenstein, 2008a, 127). En este caso, el sentido viene dado por la relación que tiene la proposición con los hechos, lo cual corresponderá a una proposición significativa. La referencia a los hechos establece el pensamiento lógico-filosófico.

Por otro, "Un pensamiento es una proposición con sentido" (Wittgenstein, 2008a, 145). Un aspecto relevante para W. consiste en concebir que la proposición tiene el carácter de figuración de los hechos del mundo razón por la cual el vienés afirma: "Una proposición es una figura de la realidad, pues si entiendo la proposición, conozco la situación que ella representa [...]" (Wittgenstein, 2008a, 150). Sin embargo, cabe advertir que la filosofía no ha abordado de modo apropiado la expresión de pensamientos de tal modo que sean acordes con una cabal comprensión de los hechos del mundo, por esta razón ciertas proposiciones denominadas filosóficas han caído en la formulación de proposiciones sinsentido (Wittgenstein, 2008a, 146).

Para el W. del Tractatus la filosofía ha de plantear los límites en la expresión de los pensamientos siempre en virtud de proposiciones que figuren el mundo de modo descriptivo, en este ámbito se destaca la importancia de la ciencia en la elaboración de proposiciones significativas (Wittgenstein, 2008a, 160). Cabe advertir, que, si bien la lógica no pertenece al campo de las ciencias naturales ${ }^{10}$, su labor permite clarificar las proposiciones, esto es, la expresión de pensamientos (Wittgenstein, 2008a, 161). W. manifiesta la posibilidad de comprender la realidad desde el análisis del lenguaje al asumir la existencia de un marco común entre el lenguaje y la realidad (Wittgenstein, 2008a, 118, 121). De esta manera, la construcción de nuestra experiencia sobre el mundo no obedece a una experiencia a priori, razón por la cual el campo del pensamiento se ordena en conformidad con la expresión de proposiciones que describen nuestra experiencia sobre los hechos y sus posibilidades, en palabras del vienés: "[...] ninguna parte de nuestra experiencia es a priori. Todo lo que vemos podría ser también de otro modo" (Wittgenstein, 2008a, 237).

\footnotetext{
${ }^{10}$ Sobre las proposiciones de las ciencias naturales en W. véase (Kenny, 1988, 94).
} 
La visión de W. es concordante con lo ya planteado por el fraile franciscano, quien articula la dependencia de las proposiciones al conocimiento intuitivo de los singulares. El pensamiento de O. en su contexto histórico, re-orienta el problema del conocimiento al campo del sentido de las proposiciones, en particular, en torno a la referencia a "estados de cosas", referencias asumidas por el lenguaje y que, tanto en $\mathrm{O}$. como en $\mathrm{W}$. comienza a desplegarse a través del aprendizaje configurado a partir del uso de términos y proposiciones. Por consiguiente, la cuestión fundamental de toda proposición con sentido obedece a su dependencia y referencia a "estados de cosas" y no a su dependencia de una verdad a priori en ambos autores.

Ahora bien, se ha sostenido que para O. y W. el problema central de los problemas asociados a la filosofía no se halla circunscrito al análisis de contenidos mentales sino al examen de nuestros términos y proposiciones, es decir, a las condiciones de posibilidad que tienen las palabras como signos en su enlace, disposición que permite la formulación de proposiciones que hacen patentes determinados "estados de cosas" (Kenny, 1988, 75). Por consiguiente, el problema central de los malos entendidos en filosofía obedece a comprender las referencias asociadas a un mundo inmutable de teorías o, también, al constante afán para la formulación de generalizaciones que escapan a la misma dinámica de la lógica del pensamiento-lenguaje la cual, según lo sostenido, sólo se articula en función de la referencia a los "estados de cosas", en este sentido decimos que nuestras proposiciones reflejan el mundo que pensamos.

Segundo, acerca de la relación pensamiento-lenguaje en O. y W. El carácter de las proposiciones en $\mathrm{O} . \mathrm{y} \mathrm{W}$. intenta, por tanto, abordar la concepción del armazón lógico del mundo abandonando la cuestión de "una" naturaleza esencial de la realidad, ambos autores en épocas distintas vuelcan su observación al análisis de un mundo fragmentado y contingente. En el caso particular de W. la proposición no pretende descansar en la expresión de "la" esencia del mundo sino abrir campo hacia una comprensión de la relación pensamiento-lenguaje $\mathrm{e}^{11}$, siempre determinada por el uso que los seres humanos damos a estos signos, signos que constituyen nuestro mundo.

\footnotetext{
${ }^{11}$ Sobre el particular véase (Kenny, 1988, 135).
} 
Ahora bien, se ha sostenido que el significado de las proposiciones descansa en el grado de referencia que tienen las mismas al momento de expresar la descripción de los hechos del mundo, esto es lo constitutivo del pensamiento. No deseamos, en este sentido, explicar ¿qué son las cosas? o ¿cuál es la estructura fundamental de la realidad? sino reconocer que la significación de nuestras proposiciones se encuentra circunscrita al orden convencional del lenguaje (Wittgenstein, 2008b, 121-122). Dentro de este campo lógico, no es posible concebir una teoría que explique el mundo, es decir, no hay una única respuesta a la totalidad de los fenómenos del mundo sino más bien la lectura del lenguaje compuesto de diversos "géneros de oraciones" (Wittgenstein, 2008b,39) o "conjuntos de proposiciones" (Ockham, 1986, 59) los cuales describen distintos estados de los singulares en directa relación con el uso del lenguaje $\mathrm{e}^{12}$. En este contexto, podemos establecer que en O. y W. la pretensión de una respuesta o explicación del mundo no concierne al orden descriptivo de las proposiciones razón por la cual su planteamiento obedece a otro horizonte que aparece en los mismos límites de la relación pensamiento-lenguaje.

El análisis de la relación pensamiento-lenguaje tendrá consecuencias fundamentales en períodos filosóficos distintos, por un lado, en el mundo medieval de $\mathrm{O}$. desde la evidente separación entre fe y razón ${ }^{13}$ la cual conlleva la manifiesta distinción entre Filosofía y Teología (Ockham, 1974, 360). Por otro, en el mundo contemporáneo de W. mediante la distinción del análisis lógico-filosófico de otras cuestiones tales como las de la Etica o la Religión ${ }^{14}$ (Wittgenstein, 2009b, 36). De este modo, para ambos pensadores, en escenarios conceptualmente distintos, el análisis filosófico se vuelca sobre una experiencia contingente de los hechos, situación que paradójicamente admite la posibilidad de un nuevo horizonte: la "trascendencia", más allá de los límites de la relación pensamiento-lenguaje. Trascendencia que, en el caso de W. debe ser considerada desde el orden de la lógica (Wittgenstein, 2008a, 241).

\footnotetext{
${ }^{12}$ Según W.: “[...] reconocemos que lo que llamamos 'proposición' y 'lenguaje’ no es una unidad formal que imaginé sino que es la familia de estructuras más o menos emparentadas entre sí" (Wittgenstein, 2008b, 121).

${ }^{13}$ Desde una perspectiva política véase esta distinción en (Ockham, 2008, 10, 154-155).

${ }^{14}$ Según Arregui, acerca de esta distinción en el Tractatus nos señala: "Si el campo del saber se limita a la objetividad homogénea de la ciencia natural, al no ser la ética una ciencia natural, pues no versa sobre los hechos, saber y ética aparecen como absolutamente heterogéneos. Saber y ética no tienen nada que ver, porque la vida queda necesariamente fuera de esa objetividad homogénea del mundo como conjunto de hechos". (Arregui, 1984, 111).
} 
Ahora bien, cabe precisar, en palabras de Anscombe, que la lógica sea trascendental no significa que las proposiciones de la lógica establezcan verdades trascendentales sino más bien que las proposiciones de la lógica muestran algo que impregna (pervades) cualquier cosa decible, sin embargo, ella es en sí misma indecible (Anscombe, 2000, 166), tal es el caso de las mismas proposiciones del Tractatus aseveradas por W. como tautológicas ${ }^{15}$. Las denominadas tautologías (proposiciones siempre verdaderas y que W. califica también como analíticas) expresan algo, pero fuera del marco de la posibilidad. En las proposiciones tautológicas no hay una descripción de hechos, sin embargo, insospechadamente, en ellas se hallan implícitas las proposiciones que dicen algo sobre los hechos mismos.

En este campo, la lectura trascendental de W. paradójicamente abre campo a lo místico ${ }^{16}$, esto es, a lo indecible desde el punto de vista lógico-filosófico, lo cual gravita en orden de proposiciones que expresan cuestiones tales como las del sentido de la vida o aquellas relacionadas con las interrogantes Eticas respecto a la naturaleza del bien o del mal. Tales proposiciones representan en sí mismas una actitud del hombre frente al mundo (Arregui, 1984, 103), considerado como un todo (Wittgenstein, 2009b, 520-521). En este contexto, es posible sostener que en $\mathrm{O}$. y W. no se plantea un rechazo a la trascendencia sino más bien, la posibilidad de proposiciones que pertenecen a un horizonte "distinto", quizás producto de una cierta "tendencia humana" a ir más allá ${ }^{17}$ de la descripción de los hechos que configuran el mundo (Wittgenstein, 2009b, 523). En esa nueva perspectiva, es posible la creación de discursos que incluyan la lectura de verdades a priori, en atención a problemáticas denominadas fundamentales del ser humano. De este modo, preguntas tales como las Eticas, que van más allá de los límites de la relación pensamien-

\footnotetext{
${ }^{15}$ Véase, además: (Anscombe, 2000, 159-160).

${ }^{16}$ Sobre el origen del término denominado "místico" en el pensador austríaco Anscombe aclara: "But Wittgenstein took the term over from Russell, who used it in a special way, with reference to an entirely ordinary feeling; one that is well expressed at 6.52: 'We feel that even if all posible scientific questions have been answered, still the problems of lif have not been touched at all'. And his further comment on this is: Of course there then just is no question left, and just is the answer"' (Anscombe, 2000, 170).

${ }^{17}$ En palabras de Wittgenstein: “[...] veo ahora que estas expresiones carentes de sentido no carecían de sentido por no haber hallado aún las expresiones correctas, sino que era su falta de sentido lo que constituía su misma esencia. Porque lo único que yo pretendía con ellas era, precisamente, ir más allá del mundo, lo cual es lo mismo que ir más allá del lenguaje significativo. Mi único propósito $-\mathrm{y}$ creo que el de todos aquellos que han tratado alguna vez de escribir o hablar de ética o religión — es arremeter contra los límites del lenguaje." (Wittgenstein, 2009b, 523)
} 
to-lenguaje, no son fruto del conocimiento intuitivo de los singulares y los hechos, sino que se vuelcan a la cuestión del mundo como un todo y sugieren su "explicación" ya en otro "horizonte". En este campo se encuentran otras problemáticas como: “[...] el primero (sujeto) por la primacía de perfección es Dios" en O. (Ockham, 1986, 58) o "De lo que no se puede hablar, hay que callar la boca"18 (Wittgenstein, 2008a, 277) en W. las cuales han de ser consideradas en virtud del reconocimiento de discursos y objetos de estudio de distinta índole a los planteados por el discurso lógico-filosófico, lo cual implica admitir cierta actitud humana que posibilita su formulación y existencia (Ockham, 1986, 57-58). En consecuencia, O. y W. detienen la mirada en la posibilidad de la configuración de órdenes diversos que no se articulan en función de la estricta relación pensamiento-lenguaje, posición que implica también el quiebre con discursos que pretenden una explicación general del mundo dentro del análisis lógico-filosófico.

Ahora bien, expuesta la posibilidad de otros horizontes en los mismos límites de la relación pensamiento-lenguaje debemos, no obstante, dar un paso atrás para volver sobre esta misma relación, y de esta manera, evaluar que entienden ambos autores por "conocimiento". Esta vez examinaremos sus posiciones en sus respectivas últimas etapas filosóficas, en ellas aparecerá una concepción de conocimiento que determinará la lectura del "pensamiento" tanto como "actividad" en O. y "operación de signos" en W.

\section{Conocimiento: "hábitos" y "usos" del lenguaje}

En las últimas etapas de las filosofías de O. y W. la lectura del conocimiento es reconocida desde la estrecha conexión entre "hábitos" y "usos" del lenguaje; en el caso de O. desde el examen del Prólogo a la Exposición sobre la Física de Aristóteles y en W. desde el Cuaderno Azul y las Investigaciones Filosóficas. El siguiente estudio tiene por objeto el análisis del conocimiento en cuanto hábitos y usos del lenguaje en ambos autores a partir del examen de

\footnotetext{
${ }^{18}$ En torno al silencio propuesto por el Tractatus Arregui nos explica: "El saber no tiene nada que ver con la praxis ética. No cabe ya reflexión alguna sobre el proyecto mundano que el lenguaje supone. No hay metalenguaje. La vida feliz, la vida buena, es la vida no problemática, la vida inmediata en la que cesa todo cuestionamiento, toda interrogación" (Arregui, 1984, 113)
} 
dos elementos: primero, respecto a la primacía del conocimiento de los singulares sobre el conocimiento abstracto, y segundo, acerca del conocimiento adquirido por hábitos y usos del lenguaje.

Primero, acerca de la primacía del conocimiento singular sobre el conocimiento abstracto. Hemos afirmado que la perspectiva de O. durante el S. XIV re-configura la noción de conocimiento al erigir como punto gravitante la fundamental búsqueda del conocimiento a partir de la estricta experiencia de los singulares y los hechos. En la posición del fraile medieval se señala la primacía del conocimiento intuitivo en cuanto conocimiento directo de los singulares y sus estados frente al denominado conocimiento abstracto de esencias, posición ampliamente desarrollada a partir de las teorías de la abstracción formal de los entes ${ }^{19}$. En este contexto, el rechazo de O. a la elaboración del conocimiento en términos de abstracción formal obedece a dos razones centrales: primero, el conocimiento en cuanto actividad intelectual depende de la unidad del intelecto, es decir, el Venerabilis Inceptor estima innecesaria la explicación de sus antecesores en torno a un conocimiento intelectual elaborado a partir de la división del entendimiento agente y posible como etapas fundamentales para la elaboración de nuestros conceptos. O. por el contrario, apela a la unidad de un solo entendimiento que obtiene sus conceptos a partir de su propia actividad intelectual desplegada a partir de la reiteración de actos mentales "hábitos" desde los cuales se configuran nuestro conocimiento como "conjuntos de hábitos" (Ockham, 1986, 51).

La actividad intelectual, por tanto, no se ejerce mediante el conocimiento de formas abstraídas de las cosas sino más bien mediante la "reiteración" de actos mentales o conocimiento habitual (Ockham, 1986, 50-51), paso fundamental para reconocer que la posición del concepto-signo se establece a partir del determinante rol de la experiencia y la "costumbre" en la denotación de nuestros términos ${ }^{20}$. Para O. la lectura del incipiente rol de la lógica circuns-

\footnotetext{
${ }^{19}$ En la filosofía escolástica del S.XIII destacan principalmente los Comentarios al De Anima de los dominicos Alberto Magno y Tomás de Aquino. Los pensadores dominicos configuran el conocimiento abstracto a la luz del pensamiento de Aristóteles, en particular, a partir de la división del intelecto en agente y posible, cuestión analizada anteriormente por los comentaristas neo-platónicos de Aristóteles entre los cuales destaca el célebre pensador árabe Avicena en el S.XI. En este escenario, el denominado proceso de abstracción de las formas por parte del intelecto agente en el hombre es posibilitado por la acción de la iluminación divina para reconocer las formas o esencias presentes en las cosas. (Tomás de Aquino 2009, $724 ; 1990,10-11)$

${ }^{20}$ Desde la Suma de la Lógica, podemos reconocer la importancia que Ockham atribuye al conocimiento
} 
crita ya al análisis de los hechos impondrá la necesaria distancia de una visión metafísica del conocimiento, posición que repercutirá en una nueva lectura de la Filosofía y su radical separación de la Teología. En este contexto, el pensador medieval reconoce el pensamiento lógico-filosófico sobre los hechos del mundo como un asunto claramente distinto a la reflexión Teológica.

Desde la posición de W. se mantiene una comprensión del conocimiento establecida en el campo del análisis de las proposiciones la cual no pretende una explicación de la actividad mental, es decir, W. a su vez también se aleja de una visión del lenguaje anclada en una visión metafísica como de cualquiera explicación circunscrita a la búsqueda de una concepción general de los términos. Por tanto, el rechazo de una posición metafísica al momento de entender el conocimiento obedece a su rechazo a un lenguaje abstracto que pretenda la "explicación" del mundo lo cual, tal como se ha mencionado, podría acontecer en el escenario de la Etica o la Teología. De esta manera, el rumbo de la filosofía desde el análisis del lenguaje reconoce la relación pensamiento-lenguaje más bien desde la descripción de los hechos que de la explicación respecto a qué es el hombre o qué es el mundo, aun cuando en la posición planteada por el Tractatus se establece el "isomorfismo lógico", esto es, la aceptación de la forma lógica del mundo a partir de la necesaria correspondencia entre la estructura de la proposición y los hechos.

En este contexto, el tema de la descripción es más patente en el W. de las Investigaciones al concebir la relación lenguaje-mundo en su sentido estrictamente pragmático en cuanto determinada por el uso en el contexto del lenguaje ordinario (Kenny, 1988, 110; Arregui, 1984, 164). Además, acerca de la descripción el mismo W. afirma: "Toda explicación tiene que desaparecer y solo la descripción ha de ocupar su lugar" (Wittgenstein, 2008b, 123). Por consiguiente, no es posible llevar el análisis del lenguaje más allá del mundo, en este sentido la filosofía no es trascendente. En atención a lo manifestado, podemos comprender que la cuestión de la primacía del conocimiento de los hechos sobre lo metafísico y abstracto es un rasgo común en las filosofías del lenguaje. El abandono de un análisis metafísico del mundo permite descu-

evidente adquirido por la experiencia de los singulares: “[...] quo apprehenso potest intellectus aliquas propositiones contingentes evidenter cognoscere [...] Unde notitia evidens unius talis singularis contingentis 'hic calor calefacit' sufficit sine aliis singularibus ad habendum notitiam evidentem de universali”'(Ockham, 1974, 522-523) 
brir la necesidad de la búsqueda de nuevas reglas que suponen la adecuada lectura del darse o no efectivo de "estados de cosas" (Wittgenstein, 2008a, 170), posición que constituye un aspecto crucial al momento de acceder a una posición descriptiva del mismo y no la urgente búsqueda de una explicación de la naturaleza de las cosas asociadas, por ejemplo, a un orden ideal (Wittgenstein, 2008b, 121).

Segundo, en torno al análisis del conocimiento como "hábitos" y "usos" del lenguaje. Se ha sostenido que $\mathrm{O}$. instala el análisis del conocimiento bajo una lectura semántica desplegada desde la intrínseca relación pensamiento-lenguaje (Ockham, 1986, 57). En el estudio de O. tiene un rol central la consideración de los alcances y límites del conocimiento el cual ha de ser comprendido como la "actividad mental" del sujeto que la ejecuta que es designada como hábitos. El hincapié del filósofo de Oxford en la comprensión del conocimiento como "actividad mental" posibilita y proyecta su sentido semántico, esto es, al establecer la intrínseca correspondencia entre "conjuntos de hábitos" con "conjuntos de proposiciones". Proposiciones que dan vida a distintos discursos en virtud de los diversos sujetos que conforman las distintas ciencias ${ }^{21}$, es decir, la formulación de distintos "conjuntos de proposiciones" que establecen progresivamente determinados conocimientos evidentes sobre el mundo. En consecuencia, diversos discursos sobre el conocimiento intuitivo de los hechos articulan las diversas ciencias (Ockham, 1986, 53). Dentro de este campo la lógica asume que el análisis del conocimiento se halla circunscrito al estricto examen del sentido de términos y proposiciones.

Por otro lado, para W. el conocimiento es posibilitado por el pensamiento como "operación de signos" lo cual se articula desde el fundamental examen de los términos, siempre en relación con el uso que les damos. Esta posición es manifiesta en la última etapa del pensamiento de W. al describir el lenguaje como "juegos" (Wittgenstein, 2008b, 331-333) postura desde la cual se describe el modo de aquello que decimos conocer. Para el vienés no hay

\footnotetext{
${ }^{21}$ En palabras de Ockham la ciencia se configura a partir de diversos conjuntos de proposiciones razón por la cual no hay un único sujeto que constituya la ciencia en cuanto conocimiento evidente: “[...] puede asentarse que la ciencia, que es una unidad de colección, no tiene un sujeto sino que, según sus diversas partes, tendrá diversos sujetos" (Ockham, 1986, 56). Por otro lado, es necesario advertir que, aún cuando Ockham en el S.XIV reconozca la ciencia como conocimiento evidente, a partir del conocimiento intuitivo de los hechos, su posición está muy lejana del concepto de ciencia desarrollado por la modernidad a partir del método científico.
} 
significados ocultos de los términos sólo la elaboración del conocimiento a partir del uso cotidiano de nuestros términos y proposiciones aprendidos por costumbre. W. muestra su posición a partir del siguiente ejemplo:

La pregunta de Sócrates ¿Qué es el conocimiento? [...] Tal como se plantea el problema, parece que algo está mal en el uso ordinario de la palabra conocimiento. Parece que no conocemos lo que significa y que, por tanto, quizá no tengamos derecho a utilizarla. Nosotros replicaríamos: 'no hay una única utilización exacta de la palabra conocimiento, pero nosotros podemos elaborar varias de estas utilizaciones, que coincidirían en un grado mayor o menor con las formas en que utiliza de hecho la palabra' (Wittgenstein, 2009a, 57)

En este sentido, abordar el análisis del lenguaje permite una aproximación distinta al problema del conocimiento al considerar el rol de los elementos que lo componen desde un punto de vista estrictamente semántico que posibilita la expresión de "estados de cosas". El conocimiento se establece en cuanto "hábitos" y "costumbres" en este sentido W. en las Investigaciones Filosóficas afirma:

No puede haber sólo una única vez en que un hombre siga una regla. No puede haber sólo una única vez en que se haga un informe, se dé una orden, o se la entienda, etc.- seguir una regla, hacer un informe, dar una orden, jugar una partida de ajedrez son costumbres (usos, instituciones). Entender una oración significa entender un lenguaje. Entender un lenguaje significa dominar una técnica (Wittgenstein, 2008b, 201).

Cabe advertir, que si bien para O. y W. el desarrollo del lenguaje y el pensamiento son aspectos estrictamente asociados a "hábitos" y "costumbres", es en $\mathrm{O}$. donde los mismos incluyen un carácter más psicológico (ipsamet intellectio), ausente en el pensamiento del primer W. pero manifiesto en el segundo, el de las Investigaciones Filosóficas. No obstante, debemos convenir que el conocimiento, para ambos pensadores, es fruto de la "actividad del pensar", la cual es comprendida por O. desde la fuerte conexión entre "conjuntos de hábitos" y "conjuntos de proposiciones" (en cuanto signos de las cosas) y por W. como "la actividad de operar con signos" (Wittgenstein, 2009a, 33). 
Es necesario reconocer, por otro lado, que el carácter del pensamiento de W. asume la cuestión del conocimiento en términos lingüísticos ya que el modo y articulación del pensamiento en la mente humana es patente a través del lenguaje (Wittgenstein, 2009a, 75). De esta manera, la relación en ambos autores en torno a la cuestión del conocimiento obedece más bien a la descripción de la misma actividad desde el análisis del lenguaje que al examen de la naturaleza del conocimiento en términos de una explicación metafísica. Para O. y W. en consecuencia, el pensamiento es descrito como una "actividad" la cual es sintetizada por W. como "operación de signos", actividad patente desde el continuo enlace y desenlace de signos, posible desde el examen de nuestros términos y proposiciones.

\section{Conclusiones}

El desafío de pensar constituye el problema radical del hombre. El avance de la técnica y sus problemáticas conlleva un ingente esfuerzo humano por trazar límites que orienten y posibiliten la comprensión de los hechos que configuran el mundo, lo cual repercute de algún modo en las cuestiones fundamentales humanas tales como la comprensión que tenemos de nosotros mismos y que representan nuestra actitud frente al mundo. En este ámbito, el examen de la relación pensamiento-lenguaje constituye un límite que se articula en función del examen de proposiciones que hacen referencia a los hechos del mundo lo cual, sin embargo, nos proyecta (en forma insospechada) fuera del mismo, posición que expone nuestra propia posición existencial fuera del horizonte lógico-filosófico. En este sentido, O. y W. serán pensadores que intentarán trazar una línea respecto a los alcances y límites de la relación pensamiento-lenguaje, lo cual incluye una determinada posición existencial frente al mismo.

Una lectura de la conexión entre las obras de O. y W. se establece desde el rol de las proposiciones en la descripción de los diversos "estados de cosas", aspecto central para el desarrollo del conocimiento evidente. La lectura de ambos pensadores señala la labor de la filosofía en torno al análisis del lenguaje, posición que marca dos giros filosóficos fundamentales en épocas y contextos culturales muy distintos. En consideración de lo anterior, reconocemos que en las reflexiones de $\mathrm{O}$. y W. tienen un rol central sus correspondientes críticas a determinados lenguajes filosóficos en que la multiplicación 
de signos abstractos eclipsa toda comprensión del mundo, en particular, a partir de la elaboración y multiplicación de discursos que imponen complejos criterios de certeza, lo cual se manifiesta en posiciones metafísicas que obstaculizan la formulación de proposiciones que muestren los hechos, es decir, los "estados de cosas". De esta manera, las soluciones planteadas por ambos pensadores gravitan en torno al detenido examen del uso de nuestros términos y proposiciones al momento de designar los singulares como los hechos que articulan nuestro mundo, por lo tanto, para O. y W. el significado comprende el fundamental rol descriptivo del lenguaje.

Para las filosofías de O. y W. además, no hay un sujeto único de las proposiciones que versan sobre los hechos y determine el "conocimiento" de los distintos fenómenos del mundo. No es posible, por tanto, abordar una explicación absoluta del mundo en el horizonte lógico-filosófico, es decir, no hay una única respuesta a la totalidad de los fenómenos del mundo sino proposiciones, "conjuntos de proposiciones" que describen los diversos estados de los singulares en directa relación con el uso del lenguaje. Una vez expuesta la lectura lógico-filosófica es manifiesta la unidad pensamiento-lenguaje.

De esta manera, es posible reconocer que el examen de los términos y proposiciones como signos nos permiten describir el mundo. Sin embargo, es posible advertir la existencia de proposiciones que cobran sentido bajo la sombra de una respuesta general del mundo, estas se hallan fuera del campo de la lógica y corresponden a otros horizontes que conllevan en sí los límites de la relación pensamiento-lenguaje. Precisamente en los límites de esta relación podemos vislumbrar y reconocer como trascendentes las preguntas fundamentales humanas asociadas a la comprensión del sentido de la vida o al examen de la misma "finalidad" de la existencia, la mayoría de estas interrogantes contenidas en la esperanza y proyección de nuestra existencia en función de nuestra actitud frente al mundo. En atención a estas consideraciones, podemos afirmar que el punto axiomático de las visiones del lenguaje en O. y W. es posibilitado por el reconocimiento del ámbito existencial en que se formulan. En este nuevo escenario, por un lado, O. afirma la existencia de un Dios desprovisto de toda idea que determine sus propios límites y, por otro, W. sostiene la existencia de proposiciones sin sentido en su Tractatus. Las visiones del conocimiento en $\mathrm{O}$. y W. nos proyectan desde la perspectiva lógica a la existencia de los bordes del mundo, su lectura como un todo. 
En definitiva, la posibilidad de respuestas a estas preguntas existenciales no forma parte de la proposición con sentido, es decir, de la relación pensamiento-lenguaje. Bajo esta singular mirada, los pensadores vislumbran que los problemas fundamentales humanos se hallan en un orden trascendente que amanece en los "límites de la expresión del pensamiento": para W. como "[...] lo que no se puede hablar" y para O. como la radical separación entre fe y razón.

\section{Referencias bibliográficas}

Anscombe GEM (2000). An introduction to Wittgenstein's Tractatus themes in the philosophy of Wittgenstein. Indiana: St Augustine's Press.

Arregui, Jorge Vicente (1984). Acción y sentido en Wittgenstein. Pamplona: EUNSA

De Andrés, Teodoro (1969). El nominalismo de Guillermo de Ockham como filosofia del lenguaje. Madrid: Ed. Gredos.

Fernández, Clemente (1980). Los filósofos medievales. Madrid: BAC.

Fortuny, Francisco José (1990). "Ontología del espíritu: principio de la epistemología de Ockham”. Convivium 33, 57-74.

Grellard, Christophe y Sang-ong-cung, Kim (2005). Le vocabulaire de Guillaume d'Ockham. Paris: Ed. Ellipses.

Hume, D. (2011). Tratado de la naturaleza humana. Madrid: Ed. Tecnos.

Kenny, Anthony (1988). Wittgenstein. Madrid: Alianza Editorial.

Ockham, Guillermo (1974). Opera Philosophica, vol. I Summa Logicae, New York: St. Bonaventure, The Franciscan Institute.

Ockham, Guillermo (1978). Opera Philosophica, vol. II Expositionis in Libros Artis Logicae Prooemium et Expositio in Librum Porphyrii de Praedicabilibus, New York: St. Bonaventure, The Franciscan Institute.

Ockham, Guillermo (1967). Opera Theologica, vol. I Scriptum in Librum Primum Sententiarum Ordinatio (Prol. et Dist.I) New York: St. Bonaventure, The Franciscan Institute. 
Ockham, Guillermo (1970). Opera Theologica, vol. II Scriptum in Librum Primum Sententiarum Ordinatio (Dist. II et III), New York: St. Bonaventure, The Franciscan Institute.

Ockham, Guillermo (1986). Los sucesivos (Prólogo a la Exposición de los ocho libros sobre la física). Barcelona: Ed. Orbis.

Ockham, Guillermo (2008). Sobre el gobierno tiránico del Papa. Madrid: Ed. Tecnos.

Panaccio, Claude (2004). Ockham on Concepts. Quebec: Ashgate.

Pannacio, Claude (1999). "Semantics and Mental Language". En Paul Vincent Spade (ed.), The Cambridge Companion to Ockham. Cambridge: Univesity Press.

Spade, Paul Vincent (1999). "Ockham's Nominalist Metaphysics: Some Main Themes”. En Paul Vincent Spade (ed.), The Cambridge Companion to Ockham. Cambridge: Univesity Press.

Tomás de Aquino (2009). Suma de Teología. Madrid: BAC.

Tomás de Aquino (1990). Perihermenias. Comentario de Santo Tomás. Valparaíso: Ed. Cerro Alegre.

Wittgenstein, Ludwig (2008a). Tractatus logico-philosophicus. Madrid: Ed. Tecnos.

Wittgenstein, Ludwig (2008b). Investigaciones Filosóficas. Barcelona: Ed. Crítica.

Wittgenstein, Ludwig (2009a). Cuadernos Azul y marrón. Madrid: Ed. Tecnos.

Wittgenstein, Ludwig (2009b). Obras completas II. Madrid: Ed. Gredos. 